\title{
Circuit et expérience de la gestion pharmaceutique des produits locaux utilisés dans un essai clinique contrôlé par un placebo
}

\author{
Mamoudou BARRY ${ }^{1,4}$, Inocent Pierre GUISSOU ${ }^{2,3,4}$, Jean Baptiste NIKIEMA ${ }^{2,4,5}$, \\ Franc ZONGO $^{3}$, Kadidjia DJIERRO $^{5}$ et Lydie BALO $^{2}$ \\ ${ }^{I}$ Centre Hospitalier Universitaire Pédiatrique Charles De Gaulle de Ouagadougou, Burkina Faso. \\ ${ }^{2}$ Unité de Formation et de Recherches en Sciences de la Santé (UFR/SDS), \\ Université de Ouagadougou, Burkina Faso. \\ ${ }^{3}$ Centre Hospitalier Universitaire Yalgado Ouedraogo de Ouagadougou, Burkina Faso. \\ ${ }^{4}$ Ecole Doctorale de Santé, Université de Ouagadougou, Burkina Faso. \\ ${ }^{5}$ Direction Générale de la Pharmacie, du Médicament et des Laboratoires du Burkina Faso, Burkina Faso. \\ *Auteur correspondant, E-mail : bmoudou@yahoo.fr; 01 BP 1198 Ouagadougou BF 01 ; \\ Tél : 0022670409640
}

\section{RESUME}

La spiruline, une algue douée de propriétés thérapeutiques et immunostimulantes, est fréquemment utilisée par les personnes infectées par le VIH (PvVIH). Un essai clinique contrôlé par un placebo, a été menée chez les personnes infectées par le VIH de type 1 (PvVIH-1) avec la spiruline enrichi en zinc et en sélénium, et appelée «spiruline plus». L'objectif de l'étude était de définir un circuit d'approvisionnement et de gestion de la « spiruline plus » et du placebo. L'étude qui a eu l'approbation du Comité d'Ethique National des Sciences et de la Santé a duré 12 mois. Les patients inclus dans l'étude ont été randomisés dans six séries. Les quatre-vingt-dix-sept patients de l'étude étaient composés de 52,6\% des sujets sous placebo contre 47,4\% sous « la spiruline plus ». Les $63.7 \%$ des patients étaient sous traitement antirétroviraux contre $36.3 \%$ sans traitement. Neuf cent cinq traitements ont été dispensés aux quatre-cent-quatre-vingt-neuf patients sous placebo et quatre cent seize patients sous «spiruline plus ». Le circuit défini a permis la dispensation de la «spiruline plus » et du placebo aux patients de l'étude pendant 12 mois.

(C) 2013 International Formulae Group. All rights reserved.

Mots clés: Circuit, gestion, «spiruline plus», PvVIH-1

\section{INTRODUCTION}

La malnutrition constitue un problème de santé publique en particulier pour les pays en voie de développement. Elle est aggravée par l'épidémie du VIH (ONUSIDA, 2010). La bonne alimentation et l'utilisation des immunostimulants ont été encouragées par l'OMS (Habib, 2008). La spiruline, une algue riche en éléments nutritifs tels que des protéines, des vitamines et des sels minéraux, a été considérée comme digne d'attention pour combattre la malnutrition (Sautier et al., 1975; Gorban et al., 2003 ; Darcas, 2004 ; Kulshreshtha et al., 2008 ; Habib, 2008). En plus de son intérêt nutritionnel, elle a des effets thérapeutiques démontrés par des études 
générales (Khan et al., 2005; Sall et al., 2009; Karkos et al., 2011) et cliniques (Branger et al., 2003; Simpore et al., 2005, 2006 ; Yamani et al., 2009 ; Anitha, 2010 ; Deng et al., 2010 ; Marcel et al., 2011 ; Selmi et al., 2011). Cependant l'aspect pharmaceutique de ces études ont été peu développé, voire inexistant. Le présent travail décrit donc l'expérience de la gestion, de la formulation, de la dispensation et le circuit de la spiruline enrichie en zinc et en sélénium («spiruline plus») dans un essai clinique contrôlé par un placebo chez les personnes infectées par le VIH-1au Burkina Faso.

\section{MATERIEL ET METHODES \\ Produits de l'essai}

La spiruline de l'étude a été produite localement à Koudougou, un centre agréé de production de la spiruline situé à $100 \mathrm{~km}$ de la capitale du Burkina Faso. Elle a été enrichie en sélénium ( $20 \mu \mathrm{g}$ / gélules) et en zinc ( $3 \mathrm{mg}$ / gélule) et appelée « spiruline plus ». Des gélules dosées à $420 \mathrm{mg}$ de cette "spiruline plus » ont été données aux patients de l'étude à la posologie de 2 gélules, 3 fois par jour au cours des repas.

Un placebo composé de farine de maïs coloré avec la chlorophylle, était donné à certains patients selon la randomisation.

Des Antirétroviraux (ARV): Les patients de l'étude ont continué à prendre les ARV qu'ils prenaient avant leur inclusion dans l'étude, en même temps que la «spiruline plus » ou le placebo.

\section{Durée et sites de l'étude}

L'étude qui a duré 12 mois a été conduite dans un centre agré de prise en charge des PvVIH : l'hôpital du jour du Centre Hospitalier Universitaire Yalgodo Ouédraogo (CHUYO) de Ouagadougou. C'est un des centres de prise en charge situé au sommet de la pyramide sanitaire du Burkina Faso.

\section{Population de l'étude}

La population de l'étude était composée de femmes et d'hommes infectés par le VIH de type 1 (PvVHI-1), âgées de 18 à 55 ans, ne souffrant pas d'une insuffisance hépatique ou rénale et résidant à Ouagadougou, capitale du Burkina Faso. Seules les femmes non enceintes ont été incluses dans l'étude.

\section{Ethique}

Le Comité d'Ethique National pour la Recherche en Santé du Burkina Faso, sur présentation d'un protocole d'étude clinique, a donné un avis favorable avant le debut de l'étude. Avant chaque inclusion, l'étude a été expliquée au participant qui, après acceptation, a signé le formulaire de consentement éclairé.

\section{Randomisation et affectation de numéro à chaque patient}

Des numéros de randomisation ont été générés par le promoteur à l'aide d'un logiciel.

Un numéro de randomisation a été affecté à chaque patient éligible qui entre dans l'étude en fonction de son statut de traitement (schéma thérapeutique), de son état immunologique (niveau des lymphocytes TCD4) et par ordre d'arrivé dans l'inclusion. Ainsi, les patients éligibles ont été répartis dans 6 séries :

- La série 200 pour les patients sous le schéma thérapeutique de deux Inhibiteurs Nucléotidiques de la Transcriptase Inverse et un Inhibiteur de Protéase (2 INTI + 1 IP) ;

- La série 300 pour les patients sous le schéma thérapeutique de deux Inhibiteurs Nucléotidiques de la Transcriptase Inverse et un Inhibiteurs Non Nucléotidiques de la Transcriptase Inverse (2 INTI + 1 INNTI) ;

- La série 400 pour les patients qui étaient en 2ème ligne de traitement antirétroviral (Abacavir, Didanosine, Lopinavir/ritonavir) ; 
- La série 600 pour les patients qui avaient leur CD4/ $\mu 1$ inférieur à $350 \mathrm{CD} 4 / \mu 1$ à l'inclusion;

la série 700 pour les patients qui avaient leur CD4/ $\mu 1$ compris entre 350 et $500 \mathrm{CD} 4 / \mu 1$ à l'inclusion ;

- La série 800 pour les patients qui avaient leur CD4 supérieurs à $500 \mathrm{CD} 4 / \mu 1$ à l'inclusion.

Dans chaque série, les patients ont été répartis dans deux groupes (groupe de patients sous «spiruline plus » et celui des patients sous placebo).

\section{Enrôlement des sujets de l'étude}

Le test de grossesse, l'urée, la créatininémie et les transaminases ont été vérifiés avant d'inclure le sujet. Des informations suivantes ont été données aux patients:

- des informations dans une langue locale et en français facile à travers des fiches d'information aux patients;

- des informations sur la gestion de la confidentialité des données, la durée de l'étude, le produit de l'étude (bénéfices et risques du produit) ;

- des conditions de participation (participation volontaire après signature du consentement ; motivation financière), les procédures et la gestion de la confidentialité et des résultats,

- des contraintes et désagréments lors des prélèvements biologiques.

Le patient qui a rempli les conditions (PvVHI-1 âgé de 18 à 55 ans, ne souffrant pas d'une insuffisance hépatique ou rénale et résidant à Ouagadougou, capitale du Burkina Faso. Seules les femmes non enceintes ont été incluses dans l'étude), a été affecté dans une des six séries.

\section{Supports utilisés}

Des fiches, des registres et un logiciel Excel ont été conçus pour le suivi et la dispensation des produits de l'étude. Des bons de commande et de transfert ont permis de commander et de recevoir la «spiruline plus » et le placebo par la pharmacie hospitalière.

\section{Circuit d'approvisionnement et gestion des produits de l'étude}

Approvisionnement en «spiruline plus » et en placebo

La Direction Générale de la Pharmacie, du Médicament et des Laboratoires (DGPML), a été au centre des activités de coordination (Figure 1). La première étape a consisté à la commande des poudres de sélénium, de zinc, de la spiruline et du placebo. Ces poudres ont été envoyées au laboratoire de l'Institut de Recherche en Sciences de la Santé (IRSS) dans sa section laboratoire de Médecine, de la Pharmacopée Traditionnelle (MEPHATRA). Une deuxième étape a permis les mises en gélules et en boîtes des produits («spiruline plus» et placebo) par ce laboratoire. Les gélules fabriquées ont été récupérées et acheminées au Laboratoire National de Santé Publique (LNSP) pour le contrôle de qualité (troisième étapes). Dans une quatrième étape, les gélules fabriquées et contrôlées été récupérées et étiquetées puis conditionnées (boîtes de la «spiruline plus» et celles du placebo) en fonction des numéros de randomisation et pour un conditionnement en quantité suffisante pour (QSP) d'un mois. La pharmacie hospitalière du Centre Hospitalier Universitaire Yalgado Ouédraogo (CHUYO) était approvisionnée proportionnellement à l'expression de besoin des produits («spiruline plus» et placebo) par le promoteur à travers des bons de commande et de transfert. La cinquième étape a consisté aux dispensations des gélules de «spiruline plus » et du placebo ainsi que les ARV.

Circuit d'approvisionnement des Antirétroviraux (ARV)

Il a été celui habituel au niveau national: La pharmacie hospitalière s'approvisionnait en ARV à partir de la Centrale d'Achat des Médicaments Génériques (CAMEG). Cette centrale est la 
structure officiellement agréé pour approvisionner tous les centres de santé surtout ceux publiques du Burkina Faso en ARV. La pharmacie hospitalière du CHUYO, stockait, gérait et dispensait par trimestre ces ARV.

\section{Suivi et réunions de l'étude}

L'étude a été dirigée par un investigateur principal et des investigateurs associés (médecins, biologistes, pharmaciens, infirmiers). Une réunion mensuelle était convoquée par l'investigateur principal autour des autres acteurs de l'étude pour discuter des problèmes rencontrés. Un comité de surveillance de la tolérance se réunissait chaque trimestre à la convocation de l'investigateur principal pour passer en revue les données de l'étude relatives à la tolérance biologique et clinique. Les sujets ont été suivis sur le plan clinique, biologique et pharmaceutique. La chaîne de froid, la gestion, la conservation, l'approvisionnement et la dispensation ont été assurés par le pharmacien hospitalier.

\section{Dispensation des produits de l'étude}

Deux principaux lots de produits ont été dispensés : le lot de la «spiruline plus » et le placebo et celui des antirétroviraux (Figure 1). Les antirétroviraux ont été délivrés aux patients en quantité suffisante (QSP) de 3 mois.

La «spiruline plus » et le placebo ont été dispensés aux patients pour une quantité suffisante d'un mois (Tableau 1). Les boîtes vides ont été retirées et les gélules restantes comptées et notées avant la dispensation de la dose mensuelle. Des questions sur la conservation des produits, les effets indésirables, les heures de prise et les rythmes de prises, ont été posées aux patients pendant la dispensation des produits. Les dispensations étaient consignées sur des supports électroniques (logiciel Excel) et papiers (registres). Les traitements non dispensés ont constitué un indicateur pour identifier les perdus de vus des patients.

\section{Analyse statistique}

Les données ont été saisies à l'aide de fichier Excel (Excel 2003). L'analyse des données a été réalisée avec le logiciel Sigma Stat (Sigma Stat 3.5). La différence de proportion entre les perdus de vu des patients sous «spiruline plus » et placebo, a été calculée en utilisant le test de Fisher.

\section{RESULTATS \\ Répartition des sujets selon le traitement et le produit de l'étude}

La population d'étude a été composée de $52,6 \%$ des sujets qui avaient pris le placebo contre $47,4 \%$ des sujets qui avaient pris la « spiruline plus ». Les patients sous traitement antirétroviraux étaient majoritaire $(63.7 \%)$ contre une minorité sans traitement $(36.3 \%)$.

\section{Dispensation.}

Le placebo ainsi que la «spiruline plus » était dispensés chaque mois. Ainsi, chaque patient qui a terminé les douze mois a eu 12 dispensations (traitements) (Tableau 1).

$\mathrm{Au}$ total, neuf cent cinq (905) traitements ont été dispensés dont quatre-centquatre-vingt-neuf (489) placebo et quatre cent seize (416) «spiruline plus » (Tableau 2).

Selon le schéma thérapeutique (Tableau 2), soixante- onze (71) trainements ont été dispensés aux patients sous 2 Inhibiteurs Nucléotidiques de la Transcriptase Inverse et une protéase (2INTI + 1IP), six cent dix-huit (618) traitements à ceux sous 2 Inhibiteurs Nucléotidiques de la Transcriptase Inverse et un Inhibiteurs Non Nucléotidiques de la Transcriptase Inverse (2INTI +1 INNTI), et cinquante-deux (52) traitements à ceux de deuxième ligne (Abacavir, Didanosine, Lopinavir/ritonavir).

Selon le niveau des CD4 au départ, soixante-dix-sept (77) traitements ont été dispensés aux patients qui ont eu leur CD4 inférieur à $350 \mathrm{CD} 4 / \mu 1$, soixante-sept (67) à ceux qui eut leur CD4 compris entre 350 et $500 \mathrm{CD} 4 / \mu 1$, et soixante-quinze (75) à ceux qui eut leur CD4 supérieur à $500 \mathrm{CD} 4 / \mu 1$ (Tableau 1). 


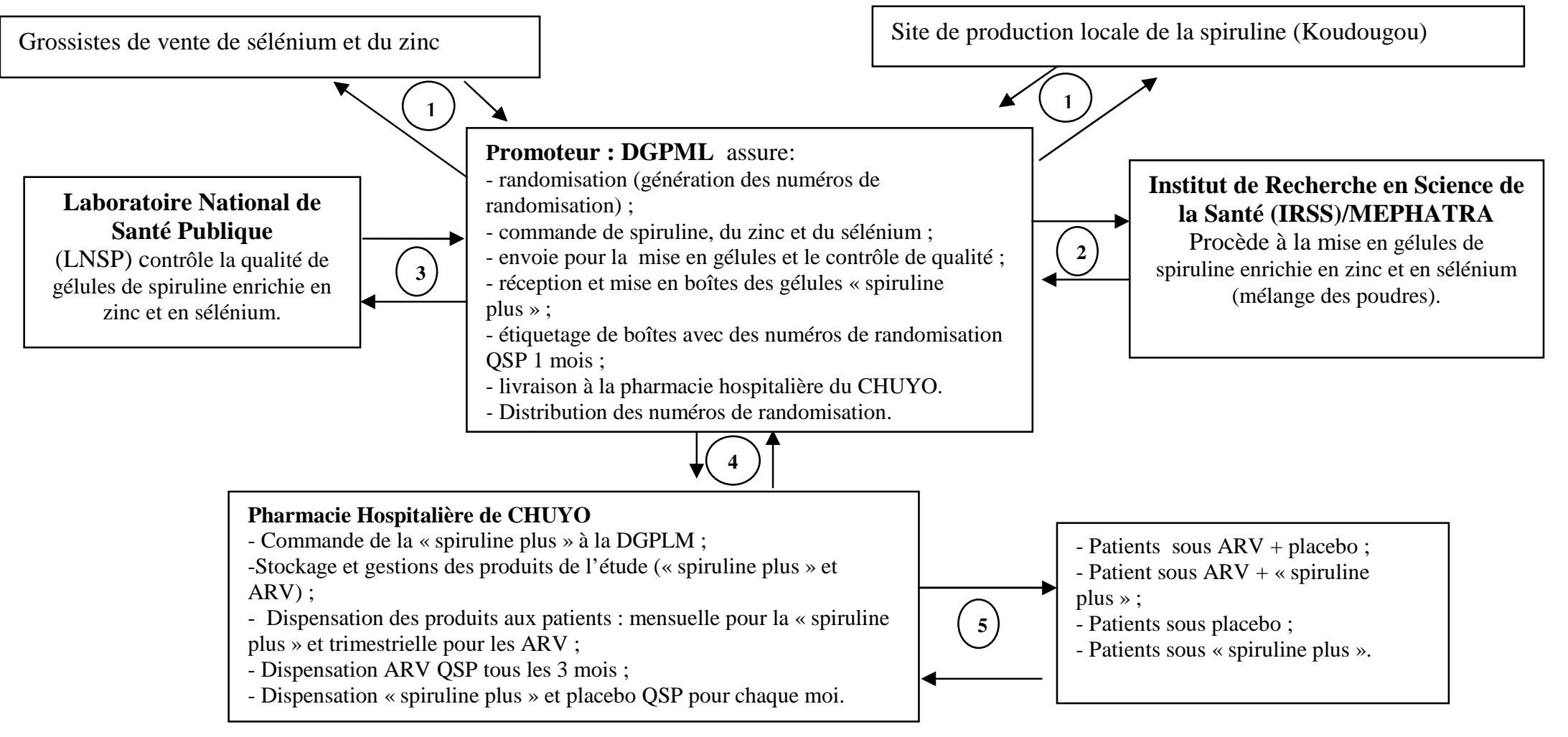

Figure 1 : Circuit de commande, de livraison et de dispensation de la spiruline enrichie en zinc et sélénium. CHUYo : Centre Hospitalier Universitaire Yalgado Ouédraogo ; DGPML : Direction Générale de la Pharmacie, du Médicament et des Laboratoires ; ARV : Antirétroviraux ; MEPHATRA : Médecine et Pharmacopée Traditionnelle. 
M. BARRY et al. / Int. J. Biol. Chem. Sci. 7(6): 2539-2548, 2013

Tableau 1 : Perdus de vue selon le fichier de la dispensation nominative des produits de l'étude.

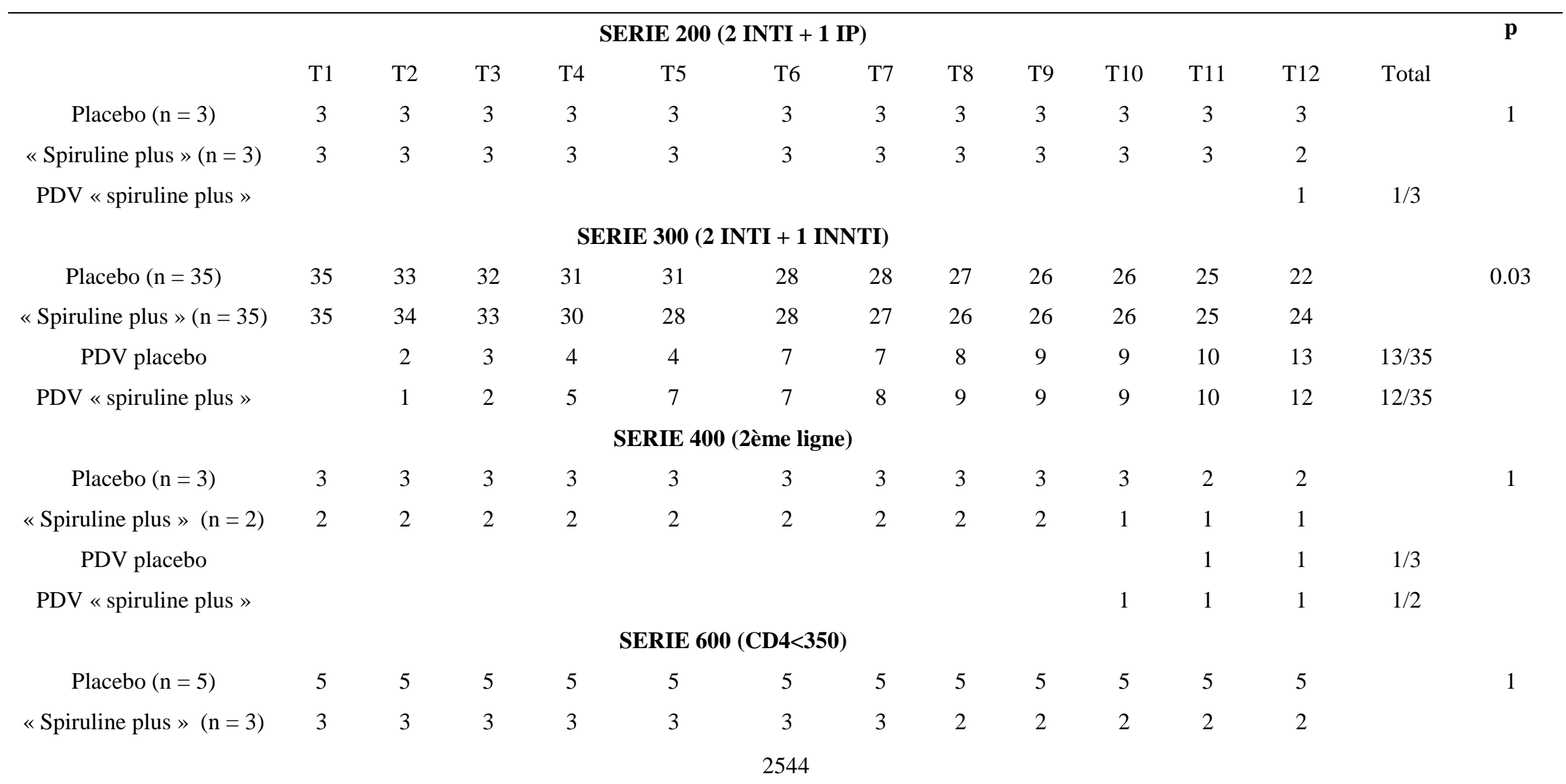


M. BARRY et al. / Int. J. Biol. Chem. Sci. 7(6): 2539-2548, 2013

PDV placebo

$\mathrm{PDV}$ « spiruline plus »

\section{Série $700(350<$ CD4<500)}

\section{Placebo}

«Spiruline plus » $(\mathrm{n}=1)$

Placebo $(\mathrm{n}=5)$

«Spiruline plus » $(\mathrm{n}=2)$

PDV placebo

$\mathrm{PDV}$ «spiruline plus »

$1 \quad 1$

(1)

Total $(\mathrm{n}=97)$

$\mathrm{T}=$ traitement $\mathrm{T} 1=$ premier traitement $=$ traitement premier mois $\quad$ INNTI Inhibiteur Non Nucléosidique de la Transcriptase Inverse

INTI = Inhibiteur Nucléosidique (ou Nucléotidique) de la Transcriptase Inverse IP = Inhibiteur de la Protéase

PDV $=$ Perdus de vus $\mathrm{p}$ : test de Ficher. 
Tableau 2 : Nombre de dispensation nominative de la «spiruline plus » ou de placebo en fonction du groupe et du schéma thérapeutique.

\begin{tabular}{|c|c|c|c|}
\hline Groupes & Sous groupes & $\begin{array}{l}\text { Conditions (Schémas } \\
\text { thérapeutiques / CD4 des } \\
\text { patients) }\end{array}$ & $\begin{array}{l}\text { Nombre de } \\
\text { dispensations }\end{array}$ \\
\hline \multirow{6}{*}{$\begin{array}{l}\text { PvVIH sous } \\
\text { ARV }\end{array}$} & \multirow[t]{3}{*}{ ARV + "spiruline plus" } & $2 \mathrm{INTI}+\mathrm{IP}$ & 35 \\
\hline & & 2INTI + INNTI & 307 \\
\hline & & $2^{\text {ème }}$ ligne & 19 \\
\hline & \multirow[t]{3}{*}{ ARV + placebo } & 2 INTI + IP & 36 \\
\hline & & 2INTI + INNTI & 311 \\
\hline & & $2^{\text {ème }}$ ligne & 33 \\
\hline \multirow{6}{*}{$\begin{array}{l}\text { PvVIH sans } \\
\text { ARV } \\
\text { au depart }\end{array}$} & \multirow[t]{3}{*}{ "spiruline plus" } & CD4 <350 au depart & 22 \\
\hline & & $350<\mathrm{CD} 4<500$ au depart & 12 \\
\hline & & CD4 > 500 au depart & 21 \\
\hline & \multirow[t]{3}{*}{ Placebo } & CD4 <350 au depart & 55 \\
\hline & & $350<\mathrm{CD} 4<500$ au depart & 0 \\
\hline & & CD4 > 500 au depart & 54 \\
\hline
\end{tabular}

Les traitements non dispensés: traitements des perdus de vu (PDV) en fonction des schémas thérapeutiques

Les traitements non dispensés ont constitué un indicateur pour identifier les perdus de vue. Au total, il y a eu trente et un (31) perdus de vue (abandons) dont quinze (15) étaient sous placebo et seize (16) sous «spiruline plus». Il n'y a pas eu de différence significative ( $\mathrm{p}>0,05)$ (Tableau 2) entre les perdus de vu de ceux qui ont pris le placebo et ceux ayant pris la «spiruline plus». Cependant il $\mathrm{y} \mathrm{a}$ eu une différence significative ( $\mathrm{p}=$ 0,03 ) entre les perdus de vue qui étaient sous le placebo et ceux sous la «spiruline plus» dans le bras des patients sous le schéma thérapeutique de 2 Inhibiteurs Nucléotidiques de la Transcriptase Inverse et un Inhibiteurs Non Nucléotidiques de la Transcriptase Inverse (2INTI + 1INNTI). Les raisons des Perdus de vue ont été multiples: exclusions de femmes qui ont eu leur test de grossesse positif au cours de suivi, abandons de sujets ayant découvert qu'ils prenaient du placebo suite aux ouvertures des gélules, abandons pour cause de retard de remboursement des frais de transport.

\section{DISCUSSION}

Des produits locaux, la «spiruline plus» et le placebo ont été fabriqués et un circuit (de commande, d'approvisionnement, de distribution et de dispensation) a été défini. Ce qui a permis de dispenser neuf cent cinq (905) traitements en une année aux quatre-vingt-dixsept (97) patients infectés par le VIH-1. La centralisation des responsabilités au niveau du promoteur a permis de garder l'esprit de double aveugle et de faire face aux difficultés.

La discussion connait des limites par absence à notre connaissance d'études similaires.

Seuls les patients sous le schéma 2 Inhibiteurs Nucléotidiques de la Transcriptase Inverse et une protéase ( 2 INTI + 1 IP), ont été nombreux à cause de la politique national 
à la période de l'étude. De façon globale, Il n'y a pas eu de différence significative ( $p>$ $0,05)$ entre les perdus de vue des patients sous placebo et sous «spiruline plus». Cependant on a noté plus de perdus de vue (abandons) chez les patients du bras placébo $(\mathrm{p}<0,05)$. Ce qui pourrait exclure le produit «spiruline plus» des causes d'abandon. On n'a pas observé une différence significative entre les deux traitements dans les autres bras.

Le nombre de patients qui ont eu leur CD4 inférieur à $350 \mathrm{CD} 4 / \mu 1$ au départ, était élevé (77) par rapport à ceux qui eut leur CD4 compris entre 350 et $500 \mathrm{CD} 4 / \mu \mathrm{l}$ (soixante-sept (67)), et à ceux qui eut leur CD4 supérieur à 500 CD4/ $\mu 1$ (soixante-quinze (75). Ce résultat témoignerait de la mise en retard des patients sous traitement à cause des dépistages tardifs.

Le circuit d'approvisionnement et de commande a été fiable mais a fait face à une lourdeur administrative qui sans la gestion particulière du pharmacien, aurait causé des ruptures de produits de l'étude. Un équipement de la pharmacie hospitalière du matériel de fabrication des produits simplifierait le circuit et diminuer la durée d'acquisition des produits de l'étude.

Les abandons pour cause de retard de payement des frais de transports, donnent à réfléchir de façon global sur des essais cliniques dans les pays en voie de développement. Cela interpelle tout promoteur à bien circonscrire les problèmes du sousdéveloppement (pauvreté mentalité...) et en tenir compte avant de commencer les études.

\section{Conclusion}

L'étude a défini un circuit et de gestion d'un complément alimentaire produit localement et utilisé dans un essai clinique contrôlé par un placebo. Le produit commandé par le promoteur est mis sous forme galénique et contrôlé. Il arrive à la pharmacie hospitalière, ou il est stocké, gérer et dispensé.

L'étude a montré une possibilité d'un essai clinique avec des produits locaux par la définition d'un circuit ainsi qu'un exemple de sa gestion. Ce qui pourra être un exemple intéressant pour d'autres études avec des produits locaux dans les essais cliniques à l'heures de la valorisation de la médecine traditionnelle dans nos pays en développement.

\section{REFERENCES}

Anitha LKC. 2010. Effect of Supplementation of Spirulina on Blood glucose, Glycosylated Hemoglobin and Lipid Profile of Male Non-Insulin Dependent Diabetics. Asian J. Exp. Biol. SCI., 1(1): 36-46.

Branger B, Cadudal JL, Delobel M, Ouoba H, Yameogo P, Ouedraogo D. 2003. Spiruline as a food supplement in case of infant malnutrition in Burkina-Faso. Arch Pediatr., 10(5): 424-431.

Darcas C. 2004. Spiruline and malnutrition. Arch Pediatr., 11(5): 466-467

Deng R, Chow T-J. 2010. Hypolipidemic, antioxidant, and antiinflammatory activities of microalgae Spirulina. Cardiovasc Ther., 28(4): e33-e45.

Gorban EN, Kuprash LP, Gorban NE. 2003. Spirulina: perspectives of the application in medicine. Lik. Sprava., (7): 100-110.

Habib MAB. 2008. Food and Agriculture Organization of the United Nations. A review on culture, production, and use of Spirulina as food for humans and feeds for domestic animals and fish. Rome, Italy: Food and Agriculture Organization of the United Nations.

Karkos PD, Leong SC, Karkos CD, Sivaji N, Assimakopoulos DA. 2011. Spirulina in clinical practice: evidence-based human applications. Evid Based Complement Alternat Med. doi: 10.1093/ecam/ nen058., 531053.

Khan Z, Bhadouria P, Bisen PS. 2005. Nutritional and therapeutic potential of Spirulina. Curr Pharm. Biotechnol., 6(5): 373-379.

Kulshreshtha A, Zacharia AJ, Jarouliya U, Bhadauriya P, Prasad GBKS, Bisen PS. 
2008. Spirulina in health care management. Curr. Pharm. Biotechnol., 9(5): 400-405.

Marcel A-K, Ekali LG, Eugene S, Arnold OE, Sandrine ED, Von der Weid D. 2011. The Effect of Spirulina platensis versus Soybean on Insulin Resistance in HIVInfected Patients: A Randomized Pilot Study. Nutrients, 3(7): 712-724.

ONUSIDA (Programme des Nations Unies sur le HIV et le SIDA). 2010. Rapport mondial rapport ONUSIDA sur l'épidémie mondiale de SIDA, ONUSIDA, Genève.

Sall MG, Dankoko B, Badiane M. 2009. Results of a nutritional rehabilitation with spirulina in Dakar. Médecine d'Afrique Noire, 46(3) : 143-146

Sautier C, Tremolieres J. 1975. Food value of the spiruline algae to man. Ann. Nutr. Aliment, 29(6): 517-534.

Selmi C, Leung PSC, Fischer L, German B, Yang C-Y, Kenny TP. 2011 The effects of Spirulina on anemia and immune function in senior citizens. Cell. Mol. Immunol., 8(3): 248-254.

Simpore J, Kabore F, Zongo F, Dansou D, Bere A, Pignatelli S. 2006. Nutrition rehabilitation of undernourished children utilizing Spiruline and Misola. Nutr J., 5: 3.

Simpore J, Zongo F, Kabore F, Dansou D, Bere A, Nikiema J-B. 2005. Nutrition rehabilitation of HIV-infected and HIVnegative undernourished children utilizing spirulina. Ann. Nutr. Metab., 49(6): 373-380.

Yamani E, Kaba-Mebri J, Mouala C, Gresenguet G, Rey JL. 2009. Use of spirulina supplement for nutritional management of HIV-infected patients: study in Bangui, Central African Republic. Med Trop., 69(1): 66-70. 